\title{
ON THE SECOND DERIVATIVES OF THE EXTREMAL-INTEGRAL
}

FOR THE INTEGRAL $\int F\left(y ; y^{\prime}\right) d t^{*}$

\author{
BY \\ ARNOLD DRESDEN
}

\section{INTRODUCTION}

It is the purpose of this paper to obtain formulas for the second derivatives of the extremal-integral arising in the theory of the integral

$$
\int F\left(y ; y^{\prime}\right) d t
$$

where $y, y^{\prime}$ are symbols for the $n$-partite numbers $\left(y_{1}, \cdots, y_{n}\right)$ and $\left(y_{1}^{\prime}\right.$, $\left.\cdots, y_{n}^{\prime}\right)$, the ' denoting differentiation with respect to the parameter $t$, expressing these derivatives in terms of "normal solutions" of the Jacobi system of differential equations, introduced into the literature by Professor Bliss. $†$ The matrix notation used in Bliss's paper will be followed here in as far as it is feasible to do so.

The methods used in this paper are analogous to those of an earlier paper; $\dagger$ for this reason only such details as are essential will be developed here. In Section 1 some preliminary facts are put together; in Section 2 the desired formulas are derived and discussed.

\section{Preliminary developments}

Using the arc length $s$ as a parameter, we denote by

$$
y=y\left(s-s_{1} ; y_{1}, y_{1}^{\prime}\right)
$$

the solutions of Euler's differential equations for our problem, which satisfy the initial conditions

$$
y\left(0 ; y_{1}, y_{1}^{\prime}\right)=y_{1}, \quad y^{\prime}\left(0 ; y_{1}, y_{1}^{\prime}\right)=y_{1}^{\prime},
$$

where $s_{1}, y_{1}\left(=y_{11}, \cdots, y_{1 n}\right)$ and $y_{1}^{\prime}\left(=\dot{y}_{11}^{\prime}, \cdots, y_{1 n}^{\prime}\right)$ are arbitrary constants of $1, n$, and $n$ dimensions respectively, subject only to the condition

${ }^{*}$ Presented to the Society, December, 1914 and September, 1916.

$\dagger$ Bliss, these Transactions, vol. 17 (1916), p. 195.

$\ddagger$ Dresden, these Trans a c tion s, vol. 17 (1916), p. 425. 
$\sum_{i=1}^{i=n} y_{1 i}^{\prime 2}=1$. The existence of such a solution follows from the general existence theorems.* Supposing that we have found an extremal passing through $A_{1}\left(a_{1}\right)$ and $A_{2}\left(a_{2}\right)$, not containing a pair of conjugate points, we can construct an extremal through the points $P_{1}\left(y_{1}\right)$ and $P_{2}\left(y_{2}\right)$ taken sufficiently near to $A_{1}$ and $A_{2}$ respectively. We find $\dagger$

$$
y=y\left(s-s_{1} ; y_{1}, \theta\left(y_{1}, y_{2}\right)\right) \equiv y(s), \quad s_{1} \leqq s \leqq s_{2},
$$

where $\theta\left(y_{1}, y_{2}\right)$ and $s_{2}\left(y_{1}, y_{2}\right)$ are determined so as to satisfy the equations

$$
\begin{aligned}
y\left(s_{2}-s_{1} ; y_{1}, \theta\right) & =y_{2}, \\
\sum_{i} \theta_{i}^{2} & =1 .
\end{aligned}
$$

The functions $y(s) \ddagger$ defined by equations (3) satisfy the following initial conditions:

$$
y\left(s_{1}\right)=y_{1}, \quad y\left(s_{2}\right)=y_{2}, \quad y^{\prime}\left(s_{1}\right)=\theta\left(y_{1}, y_{2}\right) .
$$

If we write $\phi$ for $y^{\prime}\left(s_{2}\right)$, we can obtain a second form for the equation of the extremal $P_{1} P_{2}$, viz.:

$$
y=y\left(s-s_{2} ; y_{2}, \phi\right) \equiv \bar{y}(s), \quad s_{1} \leqq s \leqq s_{2} ;
$$

the functions $\bar{y}(s)$ satisfy the conditions

$$
\bar{y}\left(s_{1}\right)=y_{1}, \quad \bar{y}\left(s_{2}\right)=y_{2}, \quad \bar{y}^{\prime}\left(s_{1}\right)=\theta, \quad \bar{y}^{\prime}\left(s_{2}\right)=\phi,
$$

while $\phi$ and $s_{1}$ satisfy the equations

$$
y\left(s_{1}-s_{2} ; y_{2}, \phi\right)=y_{1}, \quad \sum_{i} \phi_{i}^{2}=1 .
$$

Along the extremal $P_{1} P_{2}$ taken in the forms (3) or (3a), we compute the integral (1). The integral obtained in this way, known as the extremalintegral connected with the integral (1), is a function of $y_{1}$ and $y_{2}$; we will denote it by $\Gamma\left(y_{1}, y_{2}\right)$ :

$$
\Gamma\left(y_{1}, y_{2}\right)=\int_{s_{1}}^{s_{2}} F\left(y(s), y^{\prime}(s)\right) d s=\int_{s_{1}}^{s_{2}} F\left(\bar{y}(s), \bar{y}^{\prime}(s)\right) d s .
$$

If we use subscript notation for the derivatives of the function $\Gamma$

$$
\left(\Gamma_{1 i}=\partial \Gamma / \partial y_{1 i}, \quad \text { etc., } \quad \Gamma_{1 i, 2 j}=\partial^{2} \Gamma / \partial y_{1 i} \partial y_{2 j}, \quad \text { etc. }\right),
$$

* Bolza, Vorlesungen über Variationsrechnung, pp. 168-188.

$\dagger$ Bolza, loc. cit., p. 597.

$\ddagger$ Whenever this can be done without sacrificing clearness, we shall write $y(s)$ in place of $y\left(s-s_{1} ; y_{1}, \theta\left(y_{1}, y_{2}\right)\right)$, and $F(s)$ for $F\left(y(s) ; y^{\prime}(s)\right) ; \bar{y}(s)$ and $\bar{F}(s)$ will be used with analogous meanings. 
we find*

(6) $\Gamma_{1 j}=-F_{n+j}\left(y_{1} ; \theta\right)=-F_{n+j}\left(s_{1}\right), \quad \Gamma_{2 j}=F_{n+j}\left(y_{2} ; \phi\right)=F_{n+j}\left(s_{2}\right)$.

The second derivatives of the function $F$ taken along the extremal $P_{1} P_{2}$ will be denoted by the symbols, used by Bolza, $\uparrow$ viz.,

$$
\frac{\partial^{2} F}{\partial y_{i} \partial y_{k}}=P_{i k}, \quad \frac{\partial^{2} F}{\partial y_{i} \partial y_{k}^{\prime}}=Q_{i k}, \quad \frac{\partial^{2} F}{\partial y_{i}^{\prime} \partial y_{k}^{\prime}}=R_{i k} .
$$

For our further work, we introduce the following functions of $s$ :

$$
\begin{array}{ll}
\rho_{i j}=\partial y_{i}(s) / \partial y_{1 j}^{\prime}, & \sigma_{i j}=\partial y_{i}(s) / \partial y_{1 j} ; \\
\bar{\rho}_{i j}=\partial \bar{y}_{i}(s) / \partial y_{2 j}^{\prime}, & \sigma_{i j}=\partial \bar{y}_{i}(s) / \partial y_{2 j} .
\end{array}
$$

From differentiation of both sides of equations (5) and (5a) with respect to the variables $y_{1 j}$ and $y_{2 j}$, it follows that these functions satisfy the following initial conditions:

$$
\begin{array}{llll}
\text { at } s=s_{1}: \rho_{i j}=0, & \rho_{i j}^{\prime}=\delta_{i j} ; & \sigma_{i j}=\delta_{i j}, & \sigma_{i j}^{\prime}=0 ; \\
\text { at } s=s_{2}: \bar{\rho}_{i j}=0, & \bar{\rho}_{i j}^{\prime}=\delta_{i j} ; & \bar{\sigma}_{i j}=\delta_{i j}, & \bar{\sigma}_{i j}^{\prime}=0 .
\end{array}
$$

Furthermore we differentiate both sides of equations (4) and (4a) with respect to the variables $y_{1 j}$ and $y_{2 j}$, and solve the resulting equations for the derivatives of $\theta_{h}$ and $\phi_{h}$; we find:

$$
\begin{array}{ll}
\Delta\left(s_{2}\right) \frac{\partial \theta_{h}}{\partial y_{1 j}}=-D_{h j}\left(s_{2}\right), & \Delta\left(s_{2}\right) \frac{\partial \theta_{h}}{\partial y_{2 j}}=\Delta_{h j}\left(s_{2}\right), \\
\bar{\Delta}\left(s_{1}\right) \frac{\partial \phi_{h}}{\partial y_{1 j}}=\bar{\Delta}_{h j}\left(s_{1}\right), & \bar{\Delta}\left(s_{1}\right) \frac{\partial \phi_{h}}{\partial y_{2 j}}=-\bar{D}_{h j}\left(s_{1}\right) .
\end{array}
$$

Here $\Delta(s)$ and $\bar{\Delta}(s)$ are determinants of order $n+1$ given by the formulas

$$
\Delta(s)=\left|\begin{array}{ll}
y^{\prime} & \rho \\
0 & \theta
\end{array}\right|, \quad \bar{\Delta}(s)=\left|\begin{array}{ll}
y^{\prime} & \bar{\rho} \\
0 & \phi
\end{array}\right| ;
$$

$D_{h j}$ is obtained from $\Delta$ by replacing the $(h+1)$ th column by $\sigma_{i j}(i=1$, $\cdots, n), 0 ; \Delta_{h j}$ is the cofactor of $\rho_{j h}$ in $\Delta$. Similar meanings are to be attributed to $\bar{D}_{h j}$ and $\bar{\Delta}_{h j}$.

\section{The SECOND Derivatives of The EXtremal-integral}

It follows from the general theory that the functions $\rho_{i j}$ and $\sigma_{i j}$ form for every fixed $j$ a solution of Jacobi's equations; $\S$ that $y^{\prime}$ is also a solution of

* Bolza, loc. cit., p. 309.

$\dagger$ Loc. cit., p. 621.

$\ddagger$ The deductions in this section have been materially simplified by suggestions from Professor Bliss, to whom, in particular, the simple determinantal form of the final formulas is due.

$\S$ See, e. g., Hadamard, Leģons sur le calcul des variations, p. 25. 
these equations has been proved by Bliss,* who has moreover shown that to every solution $u$ of Jacobi's equations, there corresponds a unique normal solution of the form $u-\lambda y^{\prime}$, characterized by the condition

$$
\left(u-\lambda y^{\prime}\right) y^{\prime}=0, \quad s_{1} \leqq s \leqq s_{2},
$$

where $\lambda$ is an uniquely determined $n$-partite constant.

The normal solutions which correspond to the solutions $\rho_{j}$ and $\sigma_{j}$ are given by the formulas

$$
\eta_{j}=\rho_{j}-\lambda_{j} y^{\prime}, \quad \zeta_{j}=\sigma_{j}-\kappa_{j} y^{\prime},
$$

where $\lambda_{j}=y^{\prime} \rho_{j}$ and $\kappa_{j}=y^{\prime} \sigma_{j}$. From equations (8) it follows that these normal solutions satisfy the following initial conditions:

$$
\text { at } s=s_{1}, \quad \eta_{i j}=0, \quad \eta_{i j}^{\prime}=\delta_{i j}-\theta_{i} \theta_{j},
$$

and

(11) at $s=s_{1}, \quad \zeta_{i j}=\delta_{i j}-\theta_{i} \theta_{j}, \quad \zeta_{i j}^{\prime}=-y_{i}^{\prime \prime}\left(s_{1}\right) \theta_{j}-y_{j}^{\prime \prime}\left(s_{1}\right) \theta_{i}$.

From equations (10) we conclude that the functions $\eta_{i} \theta$ form for $i=1, \cdots$, $n$ a normal solution whose elements vanish at $s=s_{1}$, together with their first derivatives; consequently they vanish identically throughout the interval $\left(s_{1} s_{2}\right)$; i. e., the $n$ normal solutions $\eta_{i j}(j=1, \cdots, n)$ are connected by the linear relation

$$
\eta_{i 1} \theta_{1}+\eta_{i 2} \theta_{2}+\cdots+\eta_{i n} \theta_{n}=0 \quad(i=1, \cdots, n) .
$$

That these normal solutions do not satisfy any other linear relation becomes evident when we notice that the coefficients $c_{j}$ for such a relation would have to satisfy, in consequence of the conditions (10), the equations

$$
\sum_{j} c_{j}\left(\delta_{i j}-\theta_{i} \theta_{j}\right)=0, \quad \text { i. e., } \quad c_{i}=\theta_{i} \sum_{j} c_{j} \theta_{j} .
$$

Hence we would have either $\sum_{j} c_{j} \theta_{j}=0$, from which would follow $c_{i}=0$, or the fact that the $c_{i}$ are proportional to the $\theta_{i}$. Since there is always one non-vanishing $\theta_{j}$, there is no loss in generality, if we suppose, for simplicity of notation, $\theta_{n} \neq 0$; in this case the solutions $\eta_{1}, \cdots, \eta_{n-1}$ form a matrix of $n-1$ linearly independent solutions.

We differentiate now equations (6) and obtain

$$
\begin{gathered}
\Gamma_{1 i, 1 j}=-Q_{j i}\left(s_{1}\right)-\sum_{h} R_{i h}\left(s_{1}\right) \frac{\partial \theta_{h}}{\partial y_{1 j}}, \\
\Gamma_{1 i, 2 j}=-\sum_{h} R_{i h}\left(s_{1}\right) \frac{\partial \theta_{h}}{\partial y_{2 j}},
\end{gathered}
$$

and similar formulas for $\Gamma_{2 i, 1 j}$ and $\Gamma_{2 i, 2 j}$. Substituting equations (9) in

* Bliss, loc. cit., p. 201. 
these formulas, we can put them into the form:

$$
\Gamma_{1 i, 1 j}=-\left|\begin{array}{lll}
Q_{i j} & 0 & R_{i} \\
\sigma_{j} & y^{\prime} & \rho \\
0 & 0 & \theta
\end{array}\right| \div\left|\begin{array}{ll}
y^{\prime} & \rho \\
0 & \theta
\end{array}\right|, \quad \Gamma_{1 i, 2 j}=\left|\begin{array}{ll}
y^{\prime} & T_{i j} \\
0 & \theta
\end{array}\right| \div\left|\begin{array}{ll}
y^{\prime} & \rho \\
0 & \theta
\end{array}\right|,
$$

where the matrix $y^{\prime} T_{i j}$ is obtained from the matrix $y^{\prime} \rho$ by replacing the $j$ th row by $0, R_{i 1}, \cdots, R_{i n}$.

To reduce the solutions $\rho_{j}$ and $\sigma_{j}$, occurring in these formulas, to the corresponding normal solutions, we subtract from each of the columns containing the elements of a solution $\rho_{j}$ or $\sigma_{j}$, the column containing $y^{\prime}$ multiplied by the proper factor $\lambda_{j}$ or $\kappa_{j}$; this will replace in each of the above determinants, $\rho$ and $\sigma$ by $\eta$ and $\zeta$ respectively. Multiply now the last $n$ columns in each of these modified determinants by $\theta_{1}, \cdots, \theta_{n}$ respectively, and place the sum of the results in the $n$th column. In view of equations (12) and of the wellknown fact that $R_{i} y^{\prime}=0$, we obtain then the following

Theorem. The second derivatives of the extremal integral $\Gamma$ are given by the formulas

$$
\begin{aligned}
\Gamma_{1 i, 1 j} & =-\left|\begin{array}{lll}
Q_{j i}\left(s_{1}\right) & 0 & R_{i}\left(s_{1}\right) \\
\zeta_{j}\left(s_{2}\right) & y^{\prime}\left(s_{2}\right) & H\left(s_{2}\right)
\end{array}\right| \div\left|y^{\prime}\left(s_{2}\right) H\left(s_{2}\right)\right|, \\
\Gamma_{1 i, 2 j} & =-\sum_{h=1}^{n-1} R_{i h}\left(s_{1}\right) H_{j h}\left(s_{2}\right) \div\left|y^{\prime}\left(s_{2}\right) H\left(s_{2}\right)\right|, \\
\Gamma_{2 i, 1 j} & =\sum_{h=1}^{n-1} R_{i h}\left(s_{2}\right) \bar{H}_{j h}\left(s_{1}\right) \div\left|y^{\prime}\left(s_{1}\right) \bar{H}\left(s_{1}\right)\right|, \\
\Gamma_{2 i, 2 j} & =\left|\begin{array}{lll}
Q_{j i}\left(s_{2}\right) & 0 & R_{i}\left(s_{2}\right) \\
\zeta_{j}\left(s_{1}\right) & y^{\prime}\left(s_{1}\right) & \bar{H}\left(s_{1}\right)
\end{array}\right| \div\left|y^{\prime}\left(s_{1}\right) \bar{H}\left(s_{1}\right)\right|,
\end{aligned}
$$

where $H$ is the matrix of the $n-1$ linearly independent normal solutions $\eta_{1}, \cdots, \eta_{n-1}$ of Jacobi's equations characterized by conditions (10); $H_{j h}$ is the cofactor of $\eta_{j h}$ in $\left|y^{\prime} H\right|$; where $\bar{H}$ and $\bar{H}_{j h}$ are similarly formed from the functions $\bar{\eta}_{j h}$, obtained by normalizing the functions $\bar{\rho}_{j h}$; and where $R_{i}$ is the $(n-1)$ partite number $\left(R_{i 1}, \cdots, R_{i, n-1}\right){ }^{*}$

It still remains to show that $\Gamma_{1 i, 2 j}=\Gamma_{2 j, 1 i}$, and that $\Gamma_{1 i, 1 j}$ and $\Gamma_{2 i, 2 j}$ are symmetric in $i$ and $j$, i. e., we have to prove the following relations:

$$
\begin{aligned}
-Q_{j i}\left(s_{1}\right) \cdot\left|y^{\prime} H\right|_{s=s_{2}} & +\sum_{h=1}^{n-1} R_{i h}\left(s_{1}\right) \cdot\left|y^{\prime} Z_{h j}\right|_{s=s_{2}} \\
& =-Q_{i j}\left(s_{1}\right) \cdot\left|y^{\prime} H\right|_{s=s_{2}}+\sum_{h=1}^{n-1} R_{j h}\left(s_{1}\right) \cdot\left|y^{\prime} Z_{h i}\right|_{s=s_{2}},
\end{aligned}
$$

(B) $\quad\left|y^{\prime} \bar{H}\right|_{s=s_{1}} \cdot \sum_{h=1}^{n-1} R_{i h}\left(s_{1}\right) \cdot H_{j h}\left(s_{2}\right)+\left|y^{\prime} H\right|_{s=s_{2}} \cdot \sum_{h=1}^{n-1} R_{j h}\left(s_{2}\right) \cdot \bar{H}_{i h}\left(s_{1}\right)$,

* The choice of the particular matrix $H$ of $n-1$ linearly independent normal solutions was based upon the assumption $\theta_{n} \neq 0$; if one of the other $\theta^{\prime} \mathrm{s}$ is different from zero, an evi- 
and the relation $(\bar{A})$, obtained from $(A)$ by replacing $H$ and $Z$ by $\bar{H}$ and $\bar{Z}$ respectively and by permuting the arguments $s_{1}$ and $s_{2}$. Here $\left|y^{\prime} Z_{h j}\right|$ is obtained from $\left|y^{\prime} H\right|$ by replacing the functions $\eta_{i h}$ by $\zeta_{i j}(i=1, \cdots, n)$; and $\left|y^{\prime} \bar{Z}_{h j}\right|$ is similarly formed from $\left|y^{\prime} \bar{H}\right|$.

To this end we begin by noticing that for any two solutions of Jacobi's equations, the relation

$$
u\left[Q v+R v^{\prime}\right]-v\left[Q u+R u^{\prime}\right]=\text { constant }
$$

holds.* Applying this formula in turn for $u=\zeta_{i}, v=\eta_{j}$, and for $u=\zeta_{i}$, $v=\zeta_{j}$, and making use of equations (10) and (11), we obtain the results

and

$$
\zeta_{i}\left[Q \eta_{j}+R \eta_{j}^{\prime}\right]-\eta_{j}\left[Q \zeta_{i}+R \zeta_{i}^{\prime}\right]=R_{i j}\left(s_{1}\right),
$$

$$
\zeta_{j}\left[Q \zeta_{i}+R \zeta_{i}^{\prime}\right]-\zeta_{i}\left[Q \zeta_{j}+R \zeta_{j}^{\prime}\right]=Q_{i j}\left(s_{1}\right)-Q_{j i}\left(s_{1}\right),
$$

by means of which formula $(A)$ reduces to

$$
\left|y^{\prime} H\right| \cdot \sum_{k l} \zeta_{k j} \zeta_{l i}\left[Q_{l k}-Q_{k l}\right]+\left.\sum_{k l g} \zeta_{k j} \zeta_{l i}\left[R_{g k} H_{l}^{g}-R_{g l} H_{k}^{g}\right]\right|_{s=s_{2}}=0 ;
$$

where $H_{k}^{g}$ represents the determinant obtained from $\left|y^{\prime} H\right|$ by replacing the elements of the $k$ th row by the derivatives of the corresponding elements of the $g$ th row. This last formula follows however immediately from equation (13) if we put $u=\xi_{k}$ and $v=\xi_{l}$, where $\xi_{i g}(s), i=1, \cdots, n$, denotes the solution of Jacobi's equations obtained by replacing the $g$ th row of $\left|y^{\prime} H\right|_{s=s_{2}}$ by the $i$ th row of $\left|y^{\prime} H\right|$ and dividing the result by the former determinant; we have then $H_{g}^{l}\left(s_{2}\right)=\left|y^{\prime} H\right|_{s=s_{2}} \cdot \xi_{l g}^{\prime}\left(s_{2}\right)$. Formula $(\bar{A})$ is proved in the same way, by the use of the functions $\bar{\eta}_{j}, \bar{\zeta}_{j}$, and $\bar{\xi}_{j}$ in equation (13), while formula $(B)$ follows also from this same equation, applied to the functions $\xi_{j}$ and $\bar{\xi}_{i}$, using the values of these functions at $s_{1}$ and at $s_{2}$.

The University of Wisconsin September, 1916

dent change of notation would yield another set of formulas precisely similar to those in the text. Professor Bliss has pointed out that if an arbitrarily selected set of $n .-1$ linearly independent normal solutions is used, formulas can be deduced which are more complicated than those in the text, but which apply independently of the $\theta$ which is different from zero.

* Von Escherich, W i e ner B er i c h te, vol. 110 (1901), p. 1376; also Hadamard, loc. cit., p. 319 , and Bolza, loc. cit., p. 626 . 